Published in final edited form as:

Curr Psychiatry Rep. 2012 June ; 14(3): 188-196. doi:10.1007/s11920-012-0269-8.

\title{
Pediatric Trichotillomania
}

\author{
Julie P. Harrison and \\ Child and Adolescent OCD, Tics, Trichotillomania, and Anxiety Group (COTTAGe), University of \\ Pennsylvania School of Medicine, 3535 Market Street, Suite 600, Philadelphia, PA 19104, USA, \\ julha@upenn.edu
}

\author{
Martin E. Franklin \\ Child and Adolescent OCD, Tics, Trichotillomania, and Anxiety Group (COTTAGe), University of \\ Pennsylvania School of Medicine, 3535 Market Street, Suite 600, Philadelphia, PA 19104, USA, \\ marty@upenn.edu
}

\section{Abstract}

Trichotillomania (TTM) is an impulse control disorder characterized by chronic hair-pulling, distress, and impairment. Although the negative effects of TTM are documented and often readily evident, there remains a paucity of psychopathology and treatment research on this disorder, particularly in pediatric populations. In an effort to improve assessment of pediatric TTM, several TTM-specific instruments for youth have now been developed to reliably identify symptoms and examine related phenomenology. Instrument development has now yielded instruments to evaluate TTM and related symptoms in the context of clinical trials of youth, and the first randomized controlled trial of any treatment for pediatric TTM was recently published. Using the initial pediatric TTM studies as building blocks, future research is now needed to create a stronger body of knowledge about the relative and combined efficacy of potential interventions for TTM in youth, as well as to examine the effects of TTM phenomenology and comorbidity on treatment outcome. Dissemination efforts must also be heightened for this knowledge to best reach these vulnerable populations.

\section{Keywords}

Trichotillomania; Pediatric; Hair-pulling; Impulse control disorders; Cognitive-behavioral therapy; Habit reversal training; Pharmacotherapy; Combined treatments

\section{Introduction}

Currently classified in the $D S M-I V-T R$ as an impulse control disorder, trichotillomania (TTM) is characterized by self-induced, repeated, and often-times noticeable hair loss [1]. TTM can become severe and extremely difficult to control. Although long recognized in the medical world [2], TTM nevertheless remains sorely understudied, which has resulted in a great deal of impairment and confusion surrounding this disorder. Although the paucity of research surrounding TTM is generally disturbing, the landscape is particularly arid when it comes to pediatric samples. Fortunately, substantive progress has been made in the past decade in the study of TTM across the developmental spectrum, and new information is available to inform novel, empirically informed hypotheses about TTM phenomenology, psychopathology, and treatment. In the context of this review, the authors hope to summarize what is known already about TTM, focusing particularly on the new 
developments in pediatric TTM, and to set the stage for future research to address some of the most pressing remaining questions.

\section{Impairment and Comorbidity}

\section{Physical Impairment}

Physical damage resulting from TTM may well be underestimated, but for some sufferers, the negative effects can be extremely impairing. The most dangerous effect of TTM was first documented in 1939 in a surgical series that described hundreds of cases of trichobezoar patients [2]. Trichobezoars (ie, hairballs) are nearly solely the result of eating pulled hair (trichophagia), which occurs in a subset of individuals with TTM. These trichophagia-caused hairballs, which result because human stomachs have great difficulty digesting hair, can be present in any TTM patient with a history of eating hair and have been documented in children as young as 4 years of age [3]. In their mildest form, trichobezoars can cause heightened risk of gastrointestinal complications. In extreme cases, trichobezoars can be fatal and often require surgical procedures for removal. Additional, and likely more common, medical complications caused by TTM include skin irritation, infections, and repetitive use injuries to the hands.

\section{Emotional Impairment}

Emotional impairment is also prevalent in TTM populations, as guilt, shame, and low selfesteem run common in individuals with this disorder $[4,5]$. As TTM typically surfaces during the sensitive developmental years, the negative social implications associated with TTM can be especially disabling [6]. Studies report that TTM also moderately impairs older children and adolescents in academic and social domains [7]. From a clinical perspective, many adolescents with TTM admit to anxiety surrounding the possibility of peers discovering their bald spots. Fear of negative social evaluation is present in many of the TTM cases, and unfortunately, it appears that these concerns of peer rejection may be wellfounded. A study conducted with a sample of eighth graders revealed that after watching videos of actors playing one of three types of roles (one role was an individual with TTM, another was an individual with a tic disorder [TD], and the third role was of a healthy control), participants rated TTM and TD individuals to have much lower social acceptability than those who had neither condition [8].

\section{Comorbidity and Functional Impairment}

Comorbidities plague individuals with TTM and add to their overall distress and impairment levels. Research has revealed the following comorbidities to be the most common in TTM populations: mood disorders, anxiety disorders, substance use disorders, eating disorders [3, 9], personality disorders in adults [10], and disruptive behavior in youth [11, 12].

Additionally, dysfunction in families is commonly reported by TTM individuals who cite pulling as a main contributor to increased family disputes and secrecy. These elements can lead to a rise in stress levels for families, which can then exacerbate the TTM symptoms, which then creates a vicious cycle of triggers and hair-pulling responses [5, 13]. Other facets of impairment caused by TTM include impaired school, work, and social functioning; lowered career aspirations; and higher numbers of missed work days [14-16]. Functional impairment is also found in these populations, as hair-pulling itself is quite time consuming, as are many of the concealment methods used to hide bald areas $[17,18]$. 


\section{Phenomenology \\ Common Pulling Sites}

Individuals with TTM report bald spots in a variety of body sites, including scalp, face, arms, legs, and pubic areas. In youth TTM populations, scalp hair appears to be the most common pulling site; however, eyebrows and eyelashes were also reported as common areas to pull [7]. In the same study, only 57\% of the sample reported more than one active pulling site. The number of pulling sites seems to vary depending on developmental level, and most TTM adults appear to pull from multiple sites, with one study reporting that $97 \%$ of their adult sample reported multiple current pulling sites [19].

\section{Pulling Styles}

The absence of a comprehensive empiric foundation regarding TTM leaves clinicians in a difficult position when attempting to evaluate and treat TTM and related symptoms. Recent empiric work, however, has begun to consistently identify two distinct pulling styles: automatic and focused. In automatic pulling, individuals report little or no awareness of their pulling; however, individuals who display focused pulling are fully aware of their pulling behavior and are typically able to identify an affective trigger. Young children predominantly display automatic pulling, while adults are more likely to engage in focused pulling [20].

This observation has led to conjecture that young children, under age 10, may not have developed the necessary skills of expressiveness or emotional awareness to recognize the sensory urges that typically precede acts of hair-pulling [21]. This concept of urges that precede pulling behavior has appeared in many studies performed with TTM populations. Most participants in these studies have indicated feelings of tension or other unpleasant sensations that precede their pulling behaviors [19].

\section{Prevalence}

Comprehensive, large-scale epidemiologic studies have yet to be published, but smaller studies estimate that TTM affects $1 \%$ to $3 \%$ of college-aged individuals [22, 23]. Interestingly, relatively high rates $(11 \%-15 \%)$ of college-aged samples report at least some noncosmetic hair-pulling [24, 25]. Because this disorder is severely underresearched, rates of pediatric hair-pulling are still largely unknown [12]. Collectively, these studies indicate that mild, nondisordered hair-pulling as well as problematic hair-pulling are more common than once believed [26].

\section{Assessment}

As described above, there are numerous factors to keep in mind when assessing TTM. Comprehensive evaluations typically include collecting information on presenting symptoms, diagnostic severity, functional impairment, differential diagnosis, comorbidity, and global assessment of functioning. When making initial diagnoses, clinicians must conduct in-depth and comprehensive evaluations to gather essential information for treatment planning and evaluating changes in symptom severity.

\section{Developmental Considerations}

Depending on the developmental level of the patient, the assessment should be tailored to the individual. Typically, for younger children, parents are invited to participate in the entire diagnostic evaluation; for older adolescents, parents are not as needed and could potentially undermine the accuracy of the results. An incisive clinician should assess whether parents will be helpful or detrimental to an interview by gathering the youth's want for privacy, 
shyness level, and experience interacting with adults. If a patient does request an interview without parents, one approach is to invite parents in at the end of the interview to present a synopsis of the child's symptoms. Gathering information from multiple perspectives is important because youth and their parents typically have differing views on many subjects. These differences could serve to best inform the diagnostician about the impairment surrounding the patient's social, educational, and personal lives and could also cue the clinician in to potential treatment barriers that might revolve around privacy issues or certain family structures. Other sources of assessment data could be collected through parent and child self-report questionnaires, symptom severity scales, self-monitoring sheets, and product recording.

\section{Measures}

Within the past decade, several TTM-specific instruments have been developed and implemented that have greatly increased our ability to reliably identify key issues. These assessments have improved and furthered the field's understanding of the psychopathology of TTM, as well as treatment variables and outcomes. These assessments include the below measures:

1. Trichotillomania Diagnostic Interview (TDI) [6]: allows clinicians to rate symptoms according to each $D S M-I V$ criterion for TTM.

2. NIMH Trichotillomania Questionnaire (includes the NIMH-TSS and the NIMHTIS) [14]:

- $\quad$ The NIMH-TSS assesses for average time spent pulling, time spent pulling on the previous day, resistance to urges, resulting distress, and daily interference.

- $\quad$ The NIMH-TIS measures TTM-related impairment based on extent of distress, extent of hair loss, and interference in daily activities.

3. Trichotillomania Scale for Children-child and parent versions (TSC-C, TSC-P) [27]: helpful to gather younger children's self-reported TTM symptoms.

4. Milwaukee Inventory of Subtypes of Trichotillomania (MIST-A [for adults] and MIST-C [for children] [28, 29]: measures which type of pulling style the individual displays (either automatic or focused).

5. Massachusetts General Hospital Hairpulling Symptom Severity Scale (MGH-HS) [30]: self-report scale that assesses for urge frequency, intensity, controllability, hair-pulling frequency, resistance, and associated distress during the prior week.

6. The Premonitory Urge for Tics Scale (PUTS) [31]: a self-report questionnaire designed to assess for the presence of premonitory sensory urges common in persons with chronic TDs (CTDs) but can be adapted clinically for use with TTM and related conditions to assess various aspects of urges.

\section{Treatment}

Once a comprehensive evaluation has been completed, clinicians can use the data to inform the treatment plan. On a clinical level, reports cite an array of treatments for TTM, including cognitive and behavioral therapies, support groups, supportive counseling, hypnosis, medications, and combined approaches [7, 16]. Unfortunately, the research on TTM treatment is not well-developed, so the guide for proper treatment choice and implementation is not as strong as it needs to be. Nevertheless, clinical judgment can be used to sort out the basic decisions, such as whether TTM is primary and thus should be the focus of intervention, whether the patient has the developmental resources and motivation 
necessary to engage in more active forms of treatment (eg, cognitive-behavioral therapy [CBT]), or whether family work is needed to better set the stage for focused work on TTM symptoms themselves.

\section{Psychopharmacology}

Thus far, most studies examining pharmacotherapy for TTM have not shown medications to be efficacious in treating TTM. Selective serotonin reuptake inhibitors have not been shown to be effective treatments for pulling, but findings from a recently published randomized controlled trial in adult TTM indicate that N-acetylcysteine (NAC) may warrant further exploration as a suitable treatment option [32•]. NAC is a glutamate modulator that was found to be superior to pill placebo in a sample of adults. This compound has been shown to have a short list of side effects, and as it is not US Food and Drug Administration regulated, it is readily available to the general population in most health food stores. Additionally, given similarities between TTM and tics, neurobiological studies have examined the efficacy of atypical neuroleptics in TTM patients. Initial trials revealed that monotherapy with olanzapine, an atypical neuroleptic, was superior to pill placebo controls in adults [32•], although the side effect profile associated with atypical neuroleptics engenders caution in considering those medications as first-line treatments.

\section{Psychotherapy}

Encouraging research on psychotherapies for TTM indicate that behavioral therapy (BT) and CBT yield relatively large effect sizes in adult TTM populations $[5,7,18]$. In general, expert opinion recommends CBT, including habit reversal training (HRT), as the first line of treatment for TTM. Behavioral interventions for TTM [33] generally surround three core elements: awareness training, stimulus control, and competing response training. Awareness training will typically include techniques (eg, self-monitoring) that intend to augment patients' awareness of pulling as well as the premonitory urge that typically precedes the pulling. Stimulus control methods are vast and varied but should function as "speed bumps" aimed at minimizing the probability of pulling behavior, in which techniques are implemented to make the individual's environment less conducive to pulling. Competing response training teaches patients to engage in a behavior or movement that is physically incompatible with pulling when the urge to pull is experienced. The concept of a premonitory urge, which has been the basis for treating CTDs, also appears to be important in TTM, as most TTM study participants have reported feelings of tension or other unpleasant sensory sensations that precede and possibly lead to pulling [34••]. Originally developed by Azrin and Nunn [35], these methods comprise the active components of behavioral treatment for TTM.

Depending on the presentation of symptoms and any comorbidities, habit reversal treatments for TTM have also implemented other techniques, such as relaxation training and cognitive strategies, that are directed at reducing the dysfunctional thoughts that may precede pulling. Although these interventions appear to be efficacious for adults with TTM, they do have limitations. Relapse for patients with TTM has been shown to be common [36-39], but research is currently under way to test methods that specifically address negative emotions in attempts to augment behavioral training and HRT [40].

Additional research needs to be conducted to examine different treatment options for different pulling styles, as it is hypothesized that focused pullers versus automatic pullers require separate intervention strategies. Different affective functions might demarcate these two pulling styles, and this differentiation needs to be taken into account when devising treatment strategies. For example, automatic pulling seems to be extremely responsive to tactile antecedents, (eg, touching head with fingertips). This pulling style, which tends to 
occur outside of awareness and often during sedentary activities, may call for more behavioral techniques, with an emphasis on awareness training, stimulus control, and competing response. Focused pulling appears more responsive to affective or cognitive antecedents and might require cognitive strategies to augment the BT strategies.

A call for further research also needs to be made for a greater number of randomized clinical trials to be conducted with pediatric TTM populations. Although initial findings for behavioral intervention and HRT approaches are encouraging, questions remain surrounding the role developmental factors play in TTM psychopathology and treatment response [12]. What little work has been done on the topic of the behavioral treatment of very young children with TTM suggests that patients respond favorably to behavioral interventions $[41 \bullet \cdot]$. For example, a case study detailing a behavioral therapy intervention for TTM in a 29-month-old child reported symptom improvement [19]. However, the sample sizes for any of these preliminary studies simply cannot support confident conclusions.

The absence of evidence from randomized controlled trials conducted with pediatric samples hinders treatment development and treatment planning for perhaps the most vulnerable population of TTM sufferers. A major priority in TTM research should be to recruit and study younger samples to improve our field's understanding of TTM right around its time of onset. This focus would lead to improved methods of detecting and treating TTM earlier and in a more effective manner. The earlier clinicians can provide treatment, the less TTM individuals will be at risk for future functional impairment and debilitating comorbid disorders.

In an attempt to help build this knowledge base, Franklin and colleagues [34••] published the main findings from the first of their randomized controlled trials examining treatment for pediatric TTM and are currently conducting another trial with a more potent comparison condition. Because these studies are so far the only ones of their kind, the authors felt it prudent to provide further details of these trials in order to supply initial guides to clinical practice for TTM in youth.

\section{Pediatric Trichotillomania: A Randomized Controlled Trial of Behavior Therapy \\ Overview}

The aim of the first trial by Franklin and colleagues [34・•] was to study the feasibility of conducting a randomized controlled trial of BT for pediatric TTM and to develop strategies for patient recruitment, assessment, and treatment. This study was designed to serve as a base to provide essential information that larger future studies could utilize when investigating the efficacy of BT more fully. Two hypotheses were proposed, the first being that CBT would produce statistically significant reductions in hair-pulling symptoms at the end of the acute treatment phase (week 8), whereas minimal attention control (MAC), essentially functioning as a wait list with a potential rescue option, would not. The second hypothesis was that gains made during acute BT would be maintained through the maintenance treatment phase (week 16).

\section{Methods}

To test these hypotheses, 24 youth between the ages of 7 and 17 were randomly assigned into one of two treatment conditions, BT or MAC. To study the durability of gains in the active treatment group, the BT group received 8 weeks of active treatment in addition to a maintenance treatment phase (four visits over 8 weeks). The MAC group was designed to control for multiple, potentially confounding variables, such as participation in a study for 
TTM, minimal contact with a therapist, repeated assessments revolving around TTM, and the passage of time. After 8 weeks in MAC, the control group was offered open BT treatment. Because it was ethically responsible to offer follow-up BT for the MAC condition, no maintenance data on the MAC condition could be collected.

Information gathered in this trial included demographic characteristics, clinical characteristics, assessment of comorbid disorders, and observational data about pulling site(s). At initial assessment, TTM diagnoses were made using the Trichotillomania Diagnostic Interview [6] which assesses TTM according to the DSM-IV criteria. The initial interview also used the Anxiety Disorders Interview Schedule for Children (ADIS-C), which was used to probe for possible comorbid psychiatric disorders. The primary measure used to examine treatment outcome was the National Institute of Mental Health Trichotillomania Severity Scale (NIMH-TSS), which collects data on symptom severity according to time spent pulling, resistance to pulling, distress levels, and functional impairment [14]. During acute treatment, independent evaluators (IEs), who were blind to treatment assignment, conducted NIMH-TSS interviews at weeks 0,4 , and 8 . The Clinical Global Impression Improvement Scale (CGI-I) was used to categorize changes in symptom severity [36], which ranged from 1 to 7 , where a score of 1 equaled much improved, 4 equaled no change, and 7 equaled much worse. The CGI-I was conducted by IEs at week 4 and week 8 . As is consistent with the literature on other pediatric clinical outcome studies, the participant qualified as a successful treatment responder only if he or she received a CGI-I score of 1 or 2 from an IE at post-treatment. For the CBT condition, follow-up evaluations were conducted during the maintenance phase as well as 3 and 6 months post-treatment.

\section{Findings and Implications}

Findings were encouraging with respect to the potential clinical utility of BT. At posttreatment, the BT condition was found to be superior to the MAC condition. BT and MAC scores did not differ significantly at the start of treatment (week 0), but for BT, NIMH-TSS scores were significantly lower at week 8 than they were for MAC participants. At posttreatment, of the BT group, $75 \%$ of the participants qualified as treatment responders (CGI-I scores of 1 or 2), while $0 \%$ in the MAC group met this criterion. Moreover, acute treatment gains in BT appeared to be sustained over the course of a 9-week maintenance phase as well as throughout a naturalistic follow-up phase. Absence of evidence for relapse after BT for pediatric TTM contrasts with much of the adult literature examining BT treatment for TTM, as adult trials examining BT effectiveness for TTM have found that relapse is common after treatment discontinuation [36- 38]. The comparison of these trials for child and adult TTM populations provides preliminary support that treatment of TTM during the childhood or adolescent years may be correlated with greater durability in outcomes than treatment during adult years.

\section{Randomized Controlled Trial of Behavior Therapy for Pediatric Trichotillomania: Current Replication and Extension Study}

\section{Overview}

Encouraged by the findings of the first trial, yet fully cognizant of the limitations of that study with respect to sample size and the potency of the comparison condition, Franklin and colleagues [34••] designed a second randomized controlled trial to replicate and extend those findings. Primary outcomes and secondary analyses from the first study served as guides for the second. For instance, secondary analyses on the first randomized controlled trial indicated that children 7 to 9 years of age experienced differences in symptom reduction compared to participants who were in the 10- to 17-year-old range. Due to low statistical power in the first randomized controlled trial, no definitive conclusions could be drawn, but 
these potential results indicate that even manualized BT can be tailored to the child's developmental level in a way that is most appropriate and effective. To achieve greater statistical power, a considerably larger sample is being recruited for the current randomized controlled trial. Additionally, to control for therapist contact and psychoeducation, a more scientifically rigorous comparison condition, supportive counseling (SC), is being used. These two design modifications should help clarify whether that BT contains the major active ingredient associated with reduction in TTM and related symptoms as well as improvements in functioning.

\section{Methods}

Similar to the first randomized controlled trial, the two primary aims of the current study are to compare the efficacy of BT to a control condition (SC) in reducing TTM symptoms at post-treatment (week 8) and to examine the maintenance of BT gains through an 8-week maintenance phase (week 16). Numerous secondary aims will examine maintenance of BT gains, predictors of acute versus long-term response to BT, the relation between focused and unfocused pulling, negative affect, and treatment outcome. Additionally, exploratory analyses will investigate whether moderators, specifically demographic and clinical characteristics (eg, age, age at onset), TTM-specific factors (eg, initial severity, pulling site), psychiatric comorbidity (eg, ADIS-C/P severity scores), and family functioning (eg, Family assessment Measure [FAM]), are related to treatment outcome on the NIMH-TSS. An investigation will also be conducted to assess whether mediators (eg, treatment satisfaction, utilization of techniques, attendance, and percentage of time parent is present in sessions) are related to outcome. To accurately capture all these data, a multitude of assessments are included in the initial evaluation, during treatment, and at post-treatment. These measures include both clinician-administered and self-report assessments. The clinician-administered measures include the previously explained TDI [6] and NIMH-TQ [14]. as well as the measures below:

1. Anxiety Disorders Interview Schedule for Children (ADIS-C) [42, 43]: a semistructured interview with established psychometric properties useful for diagnosing the major $D S M-I V$ anxiety, mood, and externalizing disorders.

2. Children's Global Assessment Scale (CGAS) [44]: provides a measure of global impairment and functioning over the previous month.

3. Clinical Global Impression-Improvement scale (CGI) [45]: used to categorize the degree of change in TTM symptoms over time.

4. Photograph Measurement [46]: photographs are taken of patients' primary pulling sites, providing a concrete assessment of treatment success (not pulling).

The parent self-report measures include the previously explained TSC-P [27] as well as the following:

1. Conners Parent Rating Scales - Revised (CPRS-R) [47]: parental ratings of their child's internalizing and externalizing symptoms.

2. Family Assessment Measure (FAM) [48]: provides a measure of family functioning

3. Connors/March Developmental Questionnaire (CMDQ) [49]: provides a comprehensive developmental, educational, and treatment history of the patient and family.

The child self-report measures include the previously explained MIST-C [29], PUTS [31], TSC-C [27], and the FAM [48], as well as the below measures: 
1. Children's Depression Inventory (CDI) [50]: inventories cognitive, affective, behavioral, and interpersonal symptoms of depression.

2. Multidimensional Anxiety Scale for Children (MASC-C) [51]: assesses four broad anxiety domains-physical symptoms, social anxiety, harm avoidance, and separation/panic.

The study design includes randomization of 60 participants into 2 treatment groups: manualdriven BT or SC. The BT program consists of eight weekly 1-hour sessions, and then an 8week maintenance phase during which relapse prevention sessions are held every 2 weeks (weeks 10, 12, 14, and 16). Brief telephone contact will occur during the weeks between inperson sessions during this maintenance phase (weeks 9, 11, 13, and 15). SC implements a "Common Practices" control treatment that takes place over 8 weeks (one session per week for 1 hour each) and is designed to briefly explore various aspects of functioning related to TTM. For both groups, an assessment of TTM, other symptoms, and functional impairment are conducted on weeks 0,4 , and 8 . After week 8 , SC participants will be offered open treatment at no cost. To gather useful data, participants who complete 8 weeks of SC will be invited to participate in follow-up assessments during the maintenance phase. Treatment for BT participants includes 8 weeks of active treatment, as well as another 8 weeks of maintenance phase in which participants meet with their clinicians every other week for relapse prevention. Assessments will occur at weeks 12 and 16 during this portion of BT treatment. All study participants will then be invited back for a 3- and 6-month naturalistic follow-up evaluation. Data from these naturalistic follow-up phases of the study will provide information about the durability of BT, which will provide clinicians with recommendations for individual patients if needed, as well as grist for hypothesis generation.

\section{Conclusions}

The past decade has been a productive one with respect to TTM research, and these recent advances set the stage nicely for studies to further advance our knowledge regarding TTM's core psychopathology, TTM's underlying neurobiology, TTM's responsiveness to existing treatment, empirically informed treatment development, and dissemination of the most effective methods into community settings in which patients and their families can access them. Instrument development efforts have now yielded psychometrically acceptable measures of TTM severity and pulling styles across the developmental spectrum, which will enable researchers to document symptom severity and symptom change, and to examine what may well be a critical predictor, if not moderator, of treatment outcome, namely the preponderance of automatic versus focused pulling. More randomized controlled trials will be needed to replicate the recent findings with behavior therapy for children and adolescents, for combined treatment in adults, and for NAC, atypical neuroleptics, and opioid antagonists across the developmental spectrum. Given that the evidence from treatment studies indicates that treatment response to any of the available therapies is neither universal nor complete, it is also clear that the development of new pharmacotherapies opens up the possibility of studying how these approaches can best be combined with behavioral interventions. It will also be imperative to do in TTM what has begun in earnest in obsessive-compulsive disorder research [52-54], which is to examine how best to improve patient outcomes for those who have completed an adequate course of empirically supported treatment yet still have residual symptoms. In TTM in particular, it is also important to continue to focus on efforts to improve the durability of treatment gains given that relapse appears to be common even in those adults who have received adequate treatment and responded well to it initially. NAC is likely to be the most influential of the new treatments given initial findings regarding its efficacy and safety, as well as the interesting questions that can be raised given its mechanisms of action. Studies of its longterm efficacy and safety are also needed, as well as of its effects in younger samples. 
Because NAC appears to be both efficacious and tolerable, it stands out among the various medication candidates for direct comparison with CBT and to combined treatment in future trials.

Progress made in the past 5 years has yet to have a major impact on clinical practice with TTM outside the academic context, and this stands as the next, if not the largest, challenge still facing the field. Efforts to improve awareness of TTM must be accelerated in order to assist providers in developing knowledge, and competence in TTM and its treatment. We are pleased that patient-oriented organizations devoted to dissemination of information about anxiety disorders (eg, Anxiety Disorders Association of America) and obsessive-compulsive disorder (eg, Obsessive Compulsive Foundation) have been working with the leading patient-oriented organization in TTM (Trichotillomania Learning Center) to include more presentations about TTM to their respective memberships. Efforts such as these will be vital in getting mental health practitioners more exposure to TTM, which in turn will improve their confidence and willingness to provide empirically informed TTM treatments in their respective communities. Accordingly, it will be critical for clinically oriented TTM researchers to meet the growing demand for information and training by developing optimized models for doing so, as these efforts may well be the best way to address the major shortage of therapist expertise in TTM that is unfortunately evident in most regions in the United States as well as globally.

\section{Acknowledgments}

Dr. Franklin has received grant support from the NIMH (nos. R01 MH079377, R01 MH077197, R01 MH55126, and R21 MH61457) and the Tourette Syndrome Association.

\section{References}

1. Diagnostic and Statistical Manual of Mental Disorders. 4th edn.. Washington, DC: American Psychiatric Association; 2001. Text Revision

2. DeBakey M, Ochsner A. Bezoars and Concretions, a Comprehensive Review of the Literature with an Analysis of 303 Collected Cases and a Presentation of 8 Additional Cases. Surgery. 1938; 4:934963. and Surgery 1939,5: 132-160.

3. Lanoue JL, Arkovitz MS. Images in clinical medicine: trichobezoar in a four-year-old-girl. N Engl J Med. 2003; 348:1242. [PubMed: 12660388]

4. Soriano JL, O'Sullivan RL, Baer L, et al. Trichotillomania and self-esteem: a survey of 62 female hair pullers. J Clin Psychiatry. 1996; 57:77-82. [PubMed: 8591973]

5. Stemberger RMT, Thomas AM, Mansueto CS, Carter JG. Personal toll of trichotillomania: behavioral and interpersonal sequelae. J Anxiety Disord. 2000; 14:97-104. [PubMed: 10770238]

6. Rothbaum BO, Ninan PT. The assessment of trichotillomania. Behav Res Ther. 1994; 32:651-662. [PubMed: 8085996]

7. Franklin ME, Flessner CA, Woods DW, et al. The Child and Adolescent Trichotillomania Impact Project: descriptive psychopathology, comorbidity, functional impairment, and treatment utilization. J Dev Behav Pediatr. 2008; 29:493-500. [PubMed: 18955898]

8. Boudjouk PJ, Woods DW, Miltenberger RG, Long ES. Negative peer evaluation in adolescents: effects of tic disorders and trichotillomania. Child Fam Behav Ther. 2000; 22:17-28.

9. Christenson GA, Mackenzie TB, Mitchell JE. Characteristics of 60 adult chronic hair pullers. Am J Psychiatry. 1991; 148:365-370. [PubMed: 1992841]

10. Christenson GA, Chernoff-Clementz E, Clementz BA. Personality and clinical characteristics in patients with trichotillomania. J Clin Psychiatry. 1992; 53:407-413. [PubMed: 1459972]

11. King RA, Scahill L, Vitulano LA, Schwab-Stone M. Childhood trichotillomania: clinical phenomenology, comorbidity, and family genetics. J Am Acad Child Adolesc Psychiatry. 1995; 34:1451-1459. [PubMed: 8543512] 
12. Tolin DF, Franklin ME, Diefenbach GJ, et al. Pediatric trichotillomania: descriptive psychopathology and an open trial of cognitive-behavioral therapy. Cogn BehavTher. 2007; 36:129-144.

13. Moore PS, Franklin ME, Keuthen NJ, et al. Family functioning in pediatric trichotillomania. Child Fam Behav Ther. 2009; 31:255-269.

14. Diefenbach GJ, Tolin DF, Crocetto J, et al. Assessment of trichotillomania: a psychometric evaluation of hair-pulling scales. J Psychopathol Behav Assess. 2005; 27:169-178.

15. Seedat S, Stein DJ. Psychosocial and economic implications of trichotillomania: a pilot study in a South African sample. CNS Spectr. 1998; 3:40-43.

16. Woods DW, Flessner CA, Franklin ME, et al. Understanding and treating trichotillomania: what we know and what we don't know. Psychiatr Clin North Am. 2006; 29:487-501. [PubMed: 16650719]

17. Swedo SE, Leonard HL. Trichotillomania: an obsessive compulsive spectrum disorder? Psychiatr Clin North Am. 1992; 15:777-790. [PubMed: 1461795]

18. Wetterneck CT, Woods DW, Norberg MM, Begotka AM. The social and economic impact of trichotillomania: results from two nonreferred samples. Behavioral Interventions. 2006; 21:97109.

19. Woods DW, Flessner CA, Franklin ME, et al. Trichotillomania Scientific Advisory Board: The Trichotillomania Impact Project (TIP): exploring phenomenology, functional impairment, and treatment utilization. J Clin Psychiatry. 2006; 67:1877-1888. [PubMed: 17194265]

20. Flessner CA, Woods DW, Franklin ME, et al. Cross-sectional study of women with trichotillomania: a preliminary examination of pulling styles, severity, phenomenology, and functional impact. Child Psychiatry Hum Dev. 2009; 40:153-167. [PubMed: 18780180]

21. Freeman JB, Garcia AM, Coyne L, et al. Early childhood OCD: preliminary findings from a family based cognitive-behavioral approach. J Am Acad Child Adolesc Psychiatry. 2008; 47:593-602. [PubMed: 18356758]

22. Reeve, E. Hair pulling in children and adolescents. In: Stein, DJ.; Christenson, GA.; Hollander, E., editors. Trichotillomania. Washington, DC: American Psychiatric Press, Inc; 1999. p. 201-224.

23. Diefenbach GJ, Tolin DF, Hannan S. Trichotillomania: impact on psychosocial functioning and quality of life. Behav Res Ther. 2005; 43:869-884. [PubMed: 15896284]

24. Hajcak G, Franklin ME, Simons RF, Keuthen NJ. Hair-pulling and skin-picking in a large college sample: prevalence and relationship to affective distress and obsessive-compulsive symptoms. J Psychopathol Behav Assess. 2006; 28:179-187.

25. Rothbaum BO, Shaw L, Morris R, Ninan PT. Prevalence of trichotillomania in a college freshman population [Letter]. J Clin Psychiatry. 1993; 54:72-73. [PubMed: 8444827]

26. Adam BS, Kashani JH. Trichotillomania in children and adolescents: review of the literature and case report. Child Psychiatry Hum Dev. 1990; 20:159-167. [PubMed: 2189699]

27. Tolin DF, Diefenbach GJ, Flessner CA, et al. The trichotillomania scale for children: development and validation. Child Psychiatry Hum Dev. 2008; 39:331-349. [PubMed: 18183484]

28. Flessner CA, Conelea CA, Woods DW, et al. Styles of pulling in trichotillomania: exploring differences in symptom severity, phenomenology, and functional impact. Behav Res Ther. 2008; 46:345-357. [PubMed: 18249363]

29. Flessner CA, Woods DW, Franklin ME, et al. Styles of pulling in youth with trichotillomania: exploring differences in symptom severity, phenomenology, and comorbid psychiatric symptoms. Behav Res Ther. 2008; 46:1055-1061. [PubMed: 18675401]

30. Keuthen NJ, O'Sullivan RL, Ricciardi JN, Shera D. The Massachusetts General Hospital (MGH) hairpulling scale: I. development and factor analyses. Psychother Psychosom. 1995; 64:141-145. [PubMed: 8657844]

31. Woods DW, Piacentini JC, Himle MB, Chang S. Initial development and psychometric properties of the premonitory urge for tics scale (PUTS) in children with Tourette syndrome. J Dev Behav Pediatr. 2005; 26:397-403. [PubMed: 16344654]

32. Grant JE, Odlaug BL, Kim SW. N-acetylcysteine, a glutamate modulator, in the treatment of trichotillomania: a double-blind, placebo-controlled study. Arch Gen Psychiat. 2009; 66:756-763. 
This was a double-blind, placebo-controlled trial to determine the use of NAC in treating adults with TTM.

33. Chamberlain SR, Menzies L, Sahakian BJ, Fineberg NA. Lifting the veil on trichotillomania. Am J Psychiatry. 2007; 164:568-574. [PubMed: 17403968]

34. Franklin ME, Cahill S, Edson AL. Behavior therapy for pediatric trichotillomania: a randomized controlled trial. J Am Acad Child Adolesc Psychiatry. 2011; 50:763-771. [PubMed: 21784296] This was a randomized controlled trial of BT for pediatric TTM.

35. Azrin NH, Nunn RG. Habit-reversal: a method of eliminating nervous habits and tics. Behav Res Ther. 1973; 11:619-628. [PubMed: 4777653]

36. Schlosser S, Black DW, Blum N, Goldstein RB. The demography, phenomenology, and family history of 22 persons with compulsive hair pulling. Ann Clin Psychiatry. 1994; 6:147-152. [PubMed: 7881494]

37. Friman PC, Blum N, Rostain A. Is hair pulling benign? J Am Acad Child Adolesc Psychiatry. 1992; 31:991-992. [PubMed: 1400141]

38. Greer JM, Capecchi MR. Hoxb8 is required for normal grooming behavior in mice. Neuron. 1994; 33:23-34. [PubMed: 11779477]

39. Rothbaum BO. The behavioral treatment of trichotillomania. Behavioural Psychotherapy. 1992; 20:85-90.

40. Keuthen NJ, Rothbaum BO, Welch SS, et al. Pilot trial of dialectical behavior therapy-enhanced habit reversal for trichotillomania. Depress Anxiety. 2010; 0:1-7.

41. Franklin ME, Edson AL, Freeman JB. Behavior therapy for pediatric trichotillomania: exploring the effects of age on treatment outcome. Child Adolesc Psychiatry Ment Health. 2010; 4:18. [PubMed: 20584275] Using data from a randomized controlled trial, this report identified and examined important variables to consider when treating pediatric TRM patients.

42. Silverman, WK.; Albano, AM. Anxiety disorders interview schedule for DSM-IV: child and parent versions. San Antonio, TX: The Psychological Corporation; 1996.

43. Silverman WK, Saavedra LM, Pina AA. Test-retest reliability of anxiety symptoms and diagnoses with anxiety disorders interview schedule for DSM-IV: Child and parent versions. J Am Acad Child Psy. 2001; 40(8):937-944.

44. Shaffer D, Gould MS, Brasic J, Ambrosini P, Fisher P, Bird H, Aluwahlia S. A children's global assessment scale. Arch Gen Psychiatry. 1983; 40:1228-1231. [PubMed: 6639293]

45. Guy, W. ECDEU Assessment Manual for Psychopharmacology. Rockville, MD: National Institute for Mental Health; 1976. Clinical Global Impressions; p. 218-222.(Revised DHEW Pub)

46. Tolin, DF.; Franklin, ME.; Diefenbach, GJ. Cognitive-behavioral treatment of pediatric trichotillomania: an open trial. In: Van Minnen, A., editor. Trichotillomania: Theory and Treatment; Symposium presented to the European Association of Behavioral and Cognitive Therapies; Maastricht, The Netherlands. 2002. (Chair)

47. Conners CK, Sitarenios G, Parker JDA, Epstein JN. The revised Conners' parent rating scale (CPRS-R): Factor structure, reliability, and criterion validity. Journal of Abnormal Child Psychiatry. 1998; 26:257-268.

48. Skinner HA, Steinhauer PD, Santa-Barbara J. The family assessment measure. Can J Commun Ment Health. 1983; 2:91-103.

49. Conners, CK.; March, JS. Conners-March Developmental Questionnaire (CMDQ). North Tonawanda, NY: Multi-Health Systems, Inc; 1999.

50. Kovacs M. CDI (the Children's Depression Inventory). Psychopharmacological Bulletin. 1985; 21:995-1000.

51. March JS, Parker JD, Sullivan K, Stallings P, Conners K. The Multidimensional Anxiety Scale for Children (MASC): factor structure, reliability, and validity. J Am Acad Child Adolesc Psychiatry. 1997; 36:554-565. [PubMed: 9100431]

52. Freeman JB, Choate-Summers ML, Garcia AM, et al. The Pediatric Obsessive-Compulsive Disorder Treatment Study II: rationale, design and methods. Child Adolesc Psychiatry Ment Health. 2009; 3:4. [PubMed: 19183470] 
53. Franklin ME, Sapyta J, Freeman JB. Cognitive-behavior therapy augmentation of pharmacotherapy in pediatric obsessive compulsive disorder: the pediatric OCD treatment study II (POTS II) randomized, controlled trial. JAMA. 2011; 1224

54. Simpson HB, Foa EB, Liebowitz MR, et al. A randomized, controlled trial of cognitive-behavioral therapy for augmenting pharmacotherapy in obsessive-compulsive disorder. Am J Psychiatry. 2008; 165:621-630. [PubMed: 18316422] 